\title{
Long-term oncologic outcomes of robotic gastrectomy for gastric cancer compared with laparoscopic gastrectomy
}

\author{
Kazutaka Obama ${ }^{1,2}$ - Yoo-Min Kim ${ }^{3,4}$ - Dae Ryong Kang ${ }^{5}$ Taeil Son ${ }^{1,6,7}$ • \\ Hyoung-Il Kim ${ }^{1,6,7}$ - Sung Hoon Noh ${ }^{1,3,6,7}$ - Woo Jin Hyung ${ }^{1,3,6,7}$
}

Received: 4 November 2016/Accepted: 9 June 2017/Published online: 21 June 2017

(c) The International Gastric Cancer Association and The Japanese Gastric Cancer Association 2017

\begin{abstract}
Background Initial experiences with robotic gastrectomy (RG) for gastric cancer have demonstrated favorable shortterm outcomes, suggesting that RG is an effective alternative to laparoscopic gastrectomy (LG). However, data on long-term survival and recurrence after RG for gastric cancer have yet to be reported. The objective of this study was to assess long-term outcomes after RG compared with LG.

Methods We retrospectively evaluated 313 and 524 patients who underwent RG or LG, respectively, for gastric cancer between July 2005 and December 2009. We compared long-term outcomes using the entire and a propensity-score matched cohort.
\end{abstract}

Kazutaka Obama and Yoo-Min Kim contributed equally to this work.

Woo Jin Hyung

wjhyung@yuhs.ac

1 Department of Surgery, Yonsei University College of Medicine, 50-1 Yonsei-ro Seodaemun-gu, Seoul 120-752, Republic of Korea

2 Department of Surgery, Graduate School of Medicine, Kyoto University, Kyoto, Japan

3 Department of Surgery, Graduate School, Yonsei University College of Medicine, Seoul, Korea

4 Department of Surgery, CHA Bundang Medical Center, CHA University, Seongnam-si, Korea

5 Office of Biostatistics, Ajou University School of Medicine, Suwon, Korea

6 Gastric Cancer Center, Yonsei Cancer Center, Yonsei University Health System, Seoul, Korea

7 Robot and MIS Center, Severance Hospital, Yonsei University Health System, Seoul, Korea
Results The entire cohort analysis revealed no statistically significant differences in 5-year overall survival(OS) or relapse-free survival(RFS) $(p=0.4112$ and $p=0.8733$, respectively): $93.3 \% \quad[95 \%$ confidence interval (CI) 89.9-95.6] and 90.7\% (95\% CI, 86.9-93.5) after RG and 91.6\% (95\% CI 88.9-93.7) and 90.5\% (95\% CI 87.6-92.7) after LG, respectively; hazard ratios for death and recurrence in the robotic group were $0.828(95 \% \mathrm{CI}$, $0.528-1.299 ; \quad p=0.4119)$ and $0.968 \quad(95 \% \quad$ CI, $0.649-1.445 ; p=0.8741)$, respectively. The propensitymatched cohort analysis demonstrated no statistically significant differences for 5-year OS or RFS ( $p=0.5207$ and $p=0.2293$, respectively): $93.2 \%$ and $90.7 \%$ after RG and $94.2 \%$ and $92.6 \%$ after LG, respectively; hazard ratios for death and recurrence in the robotic group were 1.194 (95\% CI, 0.695-2.062; $p=0.5214)$ and 1.343 (95\% CI, $0.830-2.192 ; p=0.2321$ ), respectively.

Conclusion The potential technical superiority of robotic system over laparoscopy did not improve oncological outcomes after gastrectomy. Long-term oncological outcomes were not different between RG and LG. Nevertheless, robotic applications in minimally invasive gastric cancer surgery may be an oncologically safe alternative.

Keywords Robotic gastrectomy - Laparoscopic gastrectomy · Gastric cancer · Long-term outcome

\section{Introduction}

With better early postoperative outcomes, minimally invasive treatments for gastric cancer have garnered tremendous popularity over open gastrectomy. In response to demands for greater accessibility to minimally invasive surgery, laparoscopic gastrectomy has emerged as an 
established treatment for gastric cancer [1]. Nonetheless, technical difficulties and the long learning curve associated with laparoscopic gastrectomy have hindered wider application of the procedure in clinical practice $[2,3]$.

Implementation of robotic surgical systems may help overcome the technical difficulties encountered when performing laparoscopic surgery, especially for complex procedures, such as prostate, cardiac, and rectal surgery [4-6]. Accordingly, a robotic system has also been used for gastric cancer surgery. Initial results of several case series have suggested that, compared with laparoscopic gastrectomy, robotic gastrectomy reduces blood loss and hospital stay with comparable oncologic pathologic outcomes [7-9]. Surgeons seem to be able to quickly gain familiarity with and proficiency in robotic gastrectomy procedures [10-12].

With these favorable initial experiences, robotic gastrectomy for gastric cancer is regarded as an effective alternative for laparoscopic gastrectomy. However, the higher costs and longer operation time associated with robotic gastrectomy could conceivably cause surgeons to become reluctant in implementing robotic gastrectomy into clinical practice. As well, the results of a prospective multicenter comparative study indicated that robotic gastrectomy has little advantage compared with laparoscopic gastrectomy in terms of short-term surgical outcomes [13].

Moreover, studies have yet to determine the long-term outcomes of robotic gastrectomy. This lacking in long-term oncologic results of robotic gastrectomy for gastric cancer still remains a major impediment to the adoption of robotic gastrectomy. We aimed to assess the oncologic safety of robotic gastrectomy by comparing it with laparoscopic gastrectomy with respect to long-term outcomes.

\section{Methods}

\section{Patients}

Robotic gastrectomy was first performed at our institute (Severance Hospital, Yonsei University Health System) in July 2005. To evaluate long-term oncologic follow-up results, we retrospectively reviewed a prospectively collected database of gastric cancer patients to identify 862 patients who underwent robotic or laparoscopic gastrectomy between July 2005 and December 2009. Twenty-two of these patients were excluded from the analyses because of palliative gastrectomy $(n=4)$ or simultaneous surgery for cancer of another organ at the time of gastrectomy $(n=18)$. Finally, 840 patients were included in the study. The study cohort comprised 315 robotic and 525 laparoscopic gastrectomy patients. Therein, we analyzed and compared patient characteristics, operative outcomes, and postoperative complications. After excluding all postoperative mortalities ( $n=3$, two in the robotic and one in the laparoscopic group), 313 robotic and 524 laparoscopic gastrectomy patients were included in the analysis of longterm oncologic outcomes: overall survival, relapse-free survival, and recurrence patterns.

To reduce the effect of selection bias on the type of surgery and potential confounding due to the retrospective nature of the study, we used propensity-score matching to adjust for significant differences in the patients' clinicopathologic characteristics (age, clinical stage, resection extent of the stomach, depth of invasion, nodal involvement, and pathologic stage). After propensity-score matching was performed, 311 patients were included in each group for the analysis (Fig. 1).

Patients were offered the choice to undergo robotic or laparoscopic gastrectomy of histologically proven gastric adenocarcinoma that did not involve the serosal layer or the extra-perigastric lymph nodes at the time of preoperative evaluation. All tumors were staged using preoperative endoscopy, endoscopic ultrasound, and abdominopelvic computed tomography. A comprehensive and detailed explanation of the surgical and oncological risks, as well as the costs of the operations, was provided to all patients before choosing a surgery: the additional expenses for robotic surgery are not covered by the Korean National Insurance System, unlike laparoscopic surgery, which is partially covered. Each patient chose the type of surgery and provided written informed consent for surgery before operation. There was no relation with industry in the study design or analyses. This study was approved by the Institutional Review Board of Severance Hospital, Yonsei University Health System (1-2010-0066).

\section{Surgery}

To achieve oncologically safe surgery, en-bloc retrieval of the stomach with the surrounding lymph nodes was adopted for all procedures. To achieve R0 resection, the extent of resection (total or distal subtotal gastrectomy) was decided according to the tumor location. The extent of lymph node dissection, $\mathrm{D} 1+\alpha, \mathrm{D} 1+\beta$, or $\mathrm{D} 2$, was performed according to the Japanese gastric cancer treatment guidelines [14]. The da Vinci Surgical System (Intuitive Surgical Inc., Sunnyvale, CA) was used for all of the robotic gastrectomies. The procedures for robotic and laparoscopic gastrectomy with lymphadenectomy have been previously described in detail $[8,15,16]$.

\section{Follow-up, recurrence, and survival}

The same follow-up protocol was used for the prospective follow-up of each patient. The protocol itemized a physical 
examination and laboratory tests, including tumor markers, every 3 months for the first year after surgery, every 6 months for the next 4 years, and then annually thereafter. A chest X-ray, abdominopelvic computed tomography, and endoscopy were performed at least once a year. Adjuvant chemotherapy with 5-fluorouracil (5-FU)-based regimens (e.g., S-1 alone, 5-FU with cisplatin, and capecitabine with oxaliplatin) were recommended to each patient with stage II or more advanced cancer, except patients with pT3NOM0 gastric cancer.

Recurrence patterns were categorized into five groups at the time of diagnosis of recurrence: loco-regional, peritoneal, hematogenous, distant lymph node, or mixed recurrence. Peritoneal recurrence included Krukenberg's tumors. Loco-regional recurrences included tumors in the gastric bed, regional lymph nodes, anastomosis site, and remnant stomach. Recurrence in distant lymph nodes was defined as that in para-aortic or extra-abdominal lymph nodes. Patients who experienced more than one type of recurrence at the time of diagnosis were categorized as mixed recurrence.

\section{Statistical analysis}

All statistical analyses were performed using SAS version 9.2 (SAS Institute, Cary, NC, USA). Comparisons between the groups were tested with Student's $t$ test for continuous variables and Chi square test or Fisher's exact test for categorical variables as appropriate. Survival analyses were performed for the entire study cohort and separately for the propensity-score matched cohorts to adjust for significant differences in the clinicopathologic characteristics of the patients.

All patients were followed-up until death or until the last follow-up date of December 31, 2014. The survival status of all patients was confirmed. Recurrence status was not confirmed in 9 patients $(1.1 \%, 3$ robotic and 6 laparoscopic group) until 6 months before the last follow-up date. The Kaplan-Meier method was used to calculate overall and relapse-free survival, and differences between the survival curves were assessed using the log-rank test. Multivariate analyses for survival were performed using the Cox proportional hazard model. $P$ values $<0.05$ were considered statistically significant.

\section{Results}

\section{Patient characteristics for the entire cohort (Table 1)}

The mean age of the robotic group was significantly younger than that of the laparoscopic group $(54.5 \pm 12.6$ versus $59.3 \pm 11.9$, respectively, $p<0.001)$. In terms of depth of tumor invasion, nodal involvement, and pathological stage, tumors in the robotic group were more advanced compared with those in the laparoscopic group. No significant differences between the two groups were noted in gender, body mass index, tumor size, or histology.

\section{Operative outcomes for the entire cohort (Table 2)}

Robotic procedures consisted of 86 total gastrectomies (27.3\%) and 229 distal subtotal gastrectomies (72.7\%). The rate of total gastrectomy was significantly higher $(p=0.010)$ in the robotic group than the laparoscopy group (19.6\%). Radical lymphadenectomy (D1 $+\beta$ or D2) was performed in almost all of the patients in both groups without statistical difference. Mean numbers of retrieved lymph nodes were also similar between the two groups.

We did not experience any conversion to laparoscopic or open surgery in the robotic group, whereas one laparoscopic procedure $(0.2 \%)$ was converted to open surgery due to uncontrollable intraabdominal bleeding during laparoscopy. The mean operation time was significantly longer $(p<0.001)$ in the robotic group $(219 \pm 45 \mathrm{~min})$ than in the laparoscopic group $(150 \pm 41 \mathrm{~min})$. The mean estimated blood loss did not differ significantly between robotic gastrectomy $(89 \pm 146 \mathrm{ml})$ and laparoscopic gastrectomy $(102 \pm 214 \mathrm{ml}, p=0.346)$.

Postoperative complications occurred similarly between the groups $(p=0.912)$ : 38 of the 315 patients $(12.1 \%)$ in the robotic group and 62 of the 525 patients $(11.8 \%)$ in the laparoscopic group. With regard to postoperative mortality, two $(0.6 \%)$ and one $(0.2 \%)$ in-hospital deaths were recorded in the robotic and laparoscopic groups, respectively. The 2 patients in the robotic group died of intraluminal bleeding and heart failure due to mitral valve regurgitation, respectively; while the patient in the laparoscopic group died of intraabdominal bleeding.

\section{Long-term oncologic outcomes for the entire cohort}

Excluding operative mortalities, the median follow-up period was 85 months (range 60-114) for patients that survived until the cutoff date. 28 patients $(8.9 \%)$ died by the cutoff date in the robotic group, compared with 61 patients $(11.6 \%)$ in the laparoscopic group. The hazard ratio for death in the robotic group, compared with the laparoscopic group, was 0.828 (95\% confidence interval (CI) $0.528-1.299 ; p=0.4119)$. The 5 -year overall survival rate was $93.3 \%$ (95\% CI 89.9-95.6) for the robotic group and $91.6 \%$ (95\% CI 88.9-93.7) for the laparoscopic group. Kaplan-Meier curves for overall survival showed no survival differences between the two groups (log-rank $p=0.4112$; Fig. 2a). 
Table 1 Patient characteristics and clinicopathological features

\begin{tabular}{|c|c|c|c|c|c|c|}
\hline & \multicolumn{2}{|l|}{ Entire cohort } & \multirow[t]{2}{*}{$P^{\dagger}$} & \multicolumn{2}{|c|}{ Propensity-score matched cohort } & \multirow[t]{2}{*}{$P^{\dagger}$} \\
\hline & Robotic $(n=315)$ & Laparoscopic $(n=525)$ & & Robotic $(n=311)$ & Laparoscopic $(n=311)$ & \\
\hline Age (years) (range) & $54.5 \pm 12.6(24-89)$ & $59.3 \pm 11.9(24-88)$ & $<0.001^{*}$ & $54.5 \pm 12.6(24-89)$ & $54.8 \pm 12.0(24-83)$ & $0.715^{\ddagger}$ \\
\hline Sex & & & 0.510 & & & $>0.999$ \\
\hline Male & $189(60.0 \%)$ & $327(62.3 \%)$ & & $187(60.1 \%)$ & $186(59.8 \%)$ & \\
\hline Female & $126(40.0 \%)$ & $198(37.7 \%)$ & & $124(39.9 \%)$ & $125(40.2 \%)$ & \\
\hline Body mass index $\left(\mathrm{kg} / \mathrm{m}^{2}\right)$ & $23.6 \pm 3.1$ & $23.5 \pm 2.9$ & $0.670^{*}$ & $23.6 \pm 3.1$ & $23.2 \pm 2.8$ & $0.082^{*}$ \\
\hline Location & & & 0.002 & & & 0.350 \\
\hline Upper third & $49(15.6 \%)$ & $55(10.4 \%)$ & & $46(14.8 \%)$ & $41(13.2 \%)$ & \\
\hline Middle third & $107(34.0 \%)$ & $141(26.9 \%)$ & & $106(34.1 \%)$ & $93(29.9 \%)$ & \\
\hline Lower third & $159(50.5 \%)$ & $329(62.7 \%)$ & & $159(51.1 \%)$ & $177(56.9 \%)$ & \\
\hline Size $(\mathrm{mm})$ & $25.5 \pm 13.0$ & $26.1 \pm 14.6$ & $0.522^{*}$ & $25.3 \pm 13.0$ & $25.3 \pm 15.0$ & $>0.999^{*}$ \\
\hline Histology & & & 0.116 & & & 0.628 \\
\hline Differentiated & $136(43.2 \%)$ & $256(48.8 \%)$ & & $135(43.4 \%)$ & $141(45.3 \%)$ & \\
\hline Undifferentiated & $179(56.8 \%)$ & $269(50.2 \%)$ & & $176(56.6 \%)$ & $170(54.7 \%)$ & \\
\hline Metastatic LN number & $1.0 \pm 3.1$ & $0.6 \pm 2.0$ & $0.043^{*}$ & $0.6 \pm 2.4$ & $1.0 \pm 3.2$ & $0.113^{\ddagger}$ \\
\hline $\mathrm{cT}$ classification $^{\mathrm{a}}$ & & & 0.501 & & & 0.914 \\
\hline $\mathrm{T} 1$ & $231(73.3 \%)$ & $394(75.0 \%)$ & & $282(90.7 \%)$ & $285(91.6 \%)$ & \\
\hline $\mathrm{T} 2$ & $68(21.6 \%)$ & $115(21.9 \%)$ & & $27(8.7 \%)$ & $24(7.8 \%)$ & \\
\hline $\mathrm{T} 3$ & $9(2.9 \%)$ & $10(1.9 \%)$ & & $2(0.6 \%)$ & $2(0.6 \%)$ & \\
\hline $\mathrm{T} 4 \mathrm{a}$ & $7(2.2 \%)$ & $6(1.1 \%)$ & & $0(0 \%)$ & $0(0 \%)$ & \\
\hline $\mathrm{cN}$ classification ${ }^{\mathrm{a}}$ & & & 0.800 & & & $>0.999$ \\
\hline No & $255(81.0 \%)$ & $415(79.0 \%)$ & & $294(94.5 \%)$ & $295(94.9 \%)$ & \\
\hline N1 & $59(18.7 \%)$ & $108(20.6 \%)$ & & $17(5.5 \%)$ & $16(5.1 \%)$ & \\
\hline $\mathrm{N} 2$ & $1(0.3 \%)$ & $2(0.4 \%)$ & & $0(0 \%)$ & $0(0 \%)$ & \\
\hline cStage $^{\mathrm{a}}$ & & & 0.105 & & & $>0.999$ \\
\hline I & $277(87.9 \%)$ & $455(86.7 \%)$ & & $304(97.7 \%)$ & $303(97.4 \%)$ & \\
\hline II & $31(9.8 \%)$ & $66(12.6 \%)$ & & $7(2.3 \%)$ & $8(2.6 \%)$ & \\
\hline III & $7(2.2 \%)$ & $4(0.8 \%)$ & & $0(0 \%)$ & $0(0 \%)$ & \\
\hline pT classification ${ }^{a}$ & & & 0.038 & & & 0.675 \\
\hline $\mathrm{T} 1 \mathrm{a}$ & $118(37.5 \%)$ & $190(36.2 \%)$ & & $117(37.6 \%)$ & $123(39.5 \%)$ & \\
\hline $\mathrm{T} 1 \mathrm{~b}$ & $126(40.0 \%)$ & $222(42.3 \%)$ & & $126(40.5 \%)$ & $133(42.8 \%)$ & \\
\hline $\mathrm{T} 2$ & $23(7.3 \%)$ & $63(12.0 \%)$ & & $22(7.1 \%)$ & $19(6.1 \%)$ & \\
\hline $\mathrm{T} 3$ & $24(7.6 \%)$ & $24(4.5 \%)$ & & $23(7.4 \%)$ & $15(4.8 \%)$ & \\
\hline $\mathrm{T} 4 \mathrm{a}$ & $24(7.6 \%)$ & $26(5.0 \%)$ & & $22(7.4 \%)$ & $21(6.8 \%)$ & \\
\hline pN classification ${ }^{\mathrm{a}}$ & & & 0.033 & & & 0.226 \\
\hline No & $249(79.0 \%)$ & $438(83.4 \%)$ & & $246(79.1 \%)$ & $265(85.2 \%)$ & \\
\hline N1 & $34(10.7 \%)$ & $44(8.4 \%)$ & & $33(10.6 \%)$ & $26(8.4 \%)$ & \\
\hline $\mathrm{N} 2$ & $15(4.8 \%)$ & $32(6.1 \%)$ & & $15(4.8 \%)$ & $10(3.2 \%)$ & \\
\hline N3 & $17(5.5 \%)$ & $11(2.1 \%)$ & & $17(5.5 \%)$ & $10(3.2 \%)$ & \\
\hline pStage $^{a}$ & & & 0.001 & & & 0.202 \\
\hline I & $254(80.6 \%)$ & $441(84.0 \%)$ & & $252(81.0 \%)$ & $267(85.9 \%)$ & \\
\hline II & $30(9.6 \%)$ & $64(12.2 \%)$ & & $29(9.3 \%)$ & $25(8.0 \%)$ & \\
\hline III & $31(9.8 \%)$ & $20(3.8 \%)$ & & $30(9.6 \%)$ & $19(6.1 \%)$ & \\
\hline
\end{tabular}

* Values are mean \pm standard deviation

$L N$ lymph node

$\dagger$ Chi square test or Fisher's exact test except

* Student's $t$ test

${ }^{\text {a }}$ UICC classification, 7 th edition 
Table 2 Operative outcomes, postoperative recovery, and morbidity

\begin{tabular}{|c|c|c|c|c|c|c|}
\hline & \multicolumn{2}{|l|}{ Entire cohort } & \multirow[t]{2}{*}{$P^{\dagger}$} & \multicolumn{2}{|c|}{ Propensity-score matched cohort } & \multirow[t]{2}{*}{$P^{\dagger}$} \\
\hline & Robotic $(n=315)$ & Laparoscopic $(n=525)$ & & Robotic $(n=311)$ & $\begin{array}{l}\text { Laparoscopic } \\
(n=311)\end{array}$ & \\
\hline Resection extent & & & 0.010 & & & 0.783 \\
\hline Subtotal & $229(72.7 \%)$ & $422(80.4 \%)$ & & $229(73.6 \%)$ & $233(74.9 \%)$ & \\
\hline Total & $86(27.3 \%)$ & $103(19.6 \%)$ & & $82(26.4 \%)$ & $78(25.1 \%)$ & \\
\hline Extent of LN dissection & & & 0.585 & & & 0.505 \\
\hline $\mathrm{D} 1+\alpha$ & $6(1.9 \%)$ & $6(1.2 \%)$ & & $6(1.9 \%)$ & $3(1.0 \%)$ & \\
\hline $\mathrm{D} 1+\beta$ & $179(56.8 \%)$ & $310(59.0 \%)$ & & $177(56.9 \%)$ & $186(59.8 \%)$ & \\
\hline D2 & $130(41.3 \%)$ & $209(39.8 \%)$ & & $128(41.6 \%)$ & $122(39.2 \%)$ & \\
\hline Retrieved LN number & $40.1 \pm 15.4$ & $38.6 \pm 14.5$ & $0.156^{*}$ & $40.0 \pm 15.2$ & $40.1 \pm 15.2$ & $0.901^{*}$ \\
\hline Conversion & 0 & $1(0.2 \%)$ & 0.438 & 0 & $1(0.3 \%)$ & $>0.999$ \\
\hline Operation time $(\min )$ & $219 \pm 45$ & $150 \pm 41$ & $<0.001^{*}$ & $218 \pm 45$ & $149 \pm 42$ & $<0.001^{*}$ \\
\hline Estimated blood loss (ml) & $89 \pm 146$ & $102 \pm 214$ & $0.346^{*}$ & $86.9 \pm 144$ & $101.8 \pm 209.8$ & $0.357^{*}$ \\
\hline Bowel function recovery (days) & $2.8 \pm 0.8$ & $3.0 \pm 1.0$ & $0.028^{*}$ & $2.8 \pm 0.8$ & $2.9 \pm 1.0$ & $0.086^{*}$ \\
\hline Soft diet (days) & $4.3 \pm 1.8$ & $4.4 \pm 2.0$ & $0.614^{*}$ & $4.4 \pm 2.0$ & $4.3 \pm 1.8$ & $0.809^{*}$ \\
\hline Postop hospital stay (days) & $7.0 \pm 12.0$ & $7.1 \pm 8.7$ & $0.910^{*}$ & $7.2 \pm 10.0$ & $7.0 \pm 12.1$ & $0.806^{*}$ \\
\hline Postop morbidity & & & 0.912 & & & $>0.999$ \\
\hline None & $277(87.9 \%)$ & $463(88.2 \%)$ & & $275(88.4 \%)$ & $275(88.4 \%)$ & \\
\hline Present & $38(12.1 \%)$ & $62(11.8 \%)$ & & $36(11.6 \%)$ & $36(11.6 \%)$ & \\
\hline Minor & $14(4.5 \%)$ & $24(4.6 \%)$ & & $24(7.7 \%)$ & $10(3.2 \%)$ & \\
\hline Major & $24(7.6 \%)$ & $38(7.2 \%)$ & & $12(3.9 \%)$ & $26(8.4 \%)$ & \\
\hline Postop mortality & $2(0.6 \%)$ & $1(0.2 \%)$ & 0.296 & $0(0.0 \%)$ & $0(0.0 \%)$ & N/A \\
\hline
\end{tabular}

* Values are mean \pm standard deviation

$L N$ lymph node

${ }^{\dagger}$ Chi square test or Fisher's exact test except

* Student's $t$ test

By the cutoff date, 37 patients $(11.8 \%)$ in the robotic group had experienced recurrence or death, compared with 70 patients $(13.4 \%)$ in the laparoscopic group. The hazard ratio for recurrence or death in the robotic group, compared with the laparoscopic group, was 0.968 (95\% CI $0.649-1.445 ; p=0.8741)$. The 5 -year relapse-free survival rate was $90.7 \%$ (95\% CI 86.9-93.5) in the robotic group and $90.5 \%$ (95\% CI 87.6-92.7) in the laparoscopic group. Kaplan-Meier curves for relapse-free survival showed no survival differences between the two groups (log-rank $p=0.8733$; Fig. 3a). When we stratified the patient groups according to pathologic stages and analyzed overall and relapse-free survival, there were no significant differences in overall or relapse-free survival between the two groups (Figs. 2 and 3).

Recurrence was recorded in 21 of the $313(6.7 \%)$ patients in the robotic group and in 26 of the 524 (5.0\%) patients in the laparoscopic group; the difference was not statistically significant $(p=0.288)$. The recurrence patterns for these two groups were also similar. Among the 21 patients with recurrence in the robotic group, there were 9 loco-regional, 5 peritoneal, 3 hematogenous, and 4 distant lymph node recurrences. Among the 26 patients with recurrence in the laparoscopic group, there were 8 locoregional, 5 peritoneal, 6 hematogenous, 3 distant lymph nodes, and 4 mixed recurrences.

Multivariate analyses using Cox proportional hazards model to investigate whether the type of operation (robotic or laparoscopic) affected the overall and relapse-free survival revealed that age, sex, resection extent, and N-classification were independent prognostic factors for both overall and relapse-free survival. However, robotic gastrectomy was not an independent risk factor for overall and relapse-free survival $(p=0.2323$ and $p=0.7196$, respectively) (Table 3 ).

\section{Patient characteristics and operative outcomes for the propensity-score matched cohort}

To reduce the influence of selection bias for type of surgery, we used propensity-score matching to compensate for differences in baseline characteristics between the two 


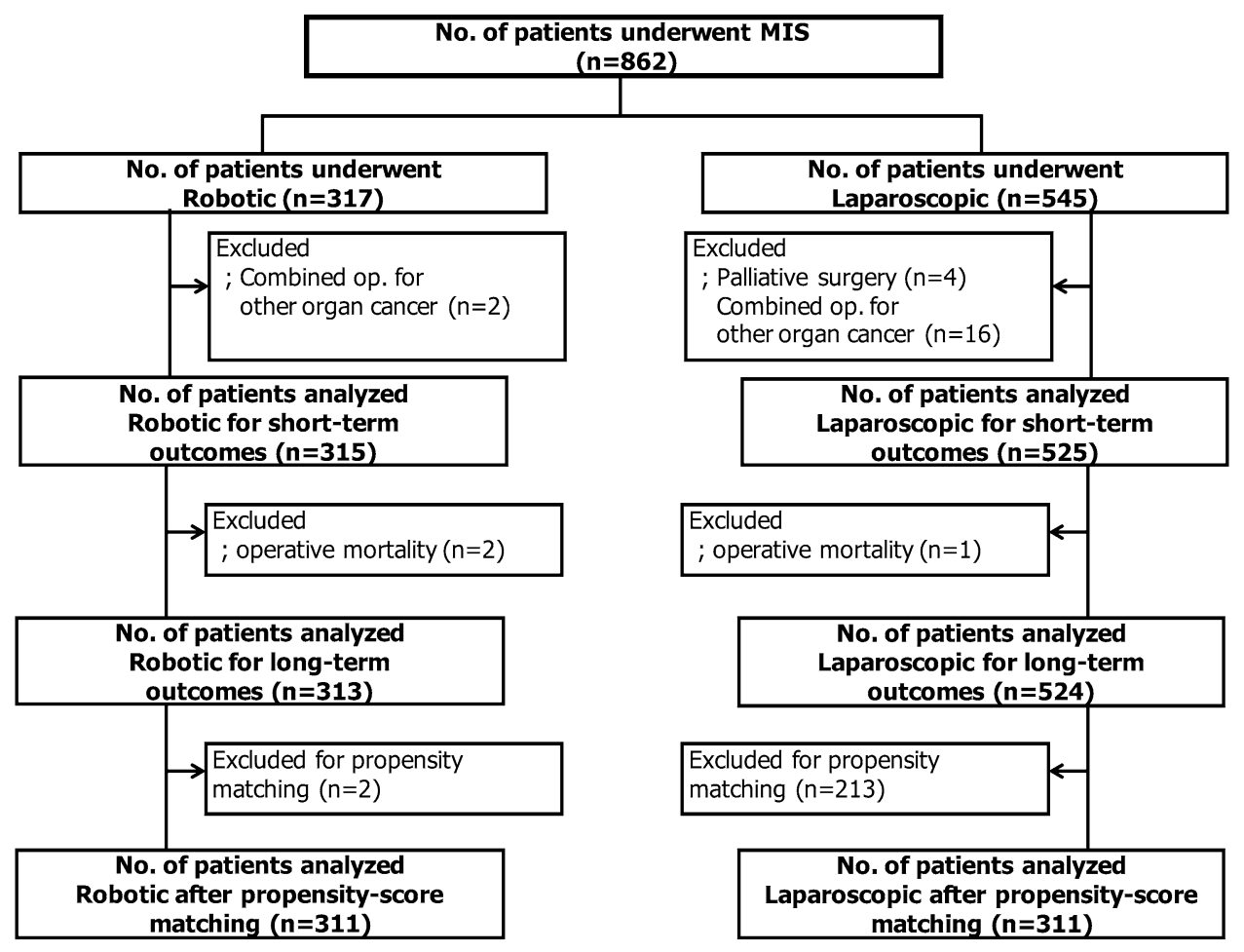

Fig. 1 Study profile

groups, since there were several statistically significant differences in clinicopathologic characteristics between the two groups. After propensity-score matching, 311 patients were included in each group. Propensity-score matching brought greater balance to the two groups (Tables 1 and 2).

In both groups, extent of gastric resection and lymphadenectomy did not differ, although the mean operation time still remained significantly longer in the robotic group $(218.0 \pm 45.0 \mathrm{~min})$ than in the laparoscopic group $(149.0 \pm 42.0 \mathrm{~min}, p<0.001)$. Clinical and pathologic results were not significantly different between the two groups. Hence, the comparability of operative outcomes and pathologic results between the groups were reproducible even after propensity-score matching to offset selection bias.

\section{Long-term oncologic outcomes for the propensity- score matched cohort}

After propensity-score matching, we analyzed overall and relapse-free survival in 311 robotic and 311 laparoscopic gastrectomy patients. $28(9.0 \%)$ patients died in the robotic group, compared with $25(8.0 \%)$ patients in the laparoscopic group. The hazard ratio for death in the robotic group, as compared with the laparoscopic group, was 1.194 (95\% CI 0.695-2.062; $p=0.5214)$. The 5-year overall survival rate was $93.2 \%$ (95\% CI 89.8-95.5) for the robotic group and $94.2 \%$ (95\% CI 91.0-96.3) for the laparoscopic group. Kaplan-Meier curves for overall survival revealed no survival differences between the two groups (log-rank $p=0.5207$; Fig. 4a).

Thirty-seven (11.9\%) patients in the robotic group experienced recurrence or death, compared with 30 patients $(9.6 \%)$ in the laparoscopic group. The hazard ratio for recurrence or death in the robotic group, as compared with the laparoscopic group, was 1.343 (95\% CI, 0.830-2.192; $p=0.2321)$. The 5-year relapse-free survival rates were 90.7\% (95\% CI 86.9-93.4) and 92.6\% (95\% CI 89.1-95.0) in the robotic and in the laparoscopic group, respectively. Kaplan-Meier curves for relapse-free survival revealed no survival differences between the two groups (log-rank $p=0.2293$; Fig. 4b).

\section{Discussion}

The clinical value of robotic application on prognosis of gastric cancer patients has been controversial. In this retrospective study, we found no survival differences in overall and relapse-free survival between robotic and laparoscopic gastrectomies for gastric cancer. The recurrence patterns were also similar between the two groups. 

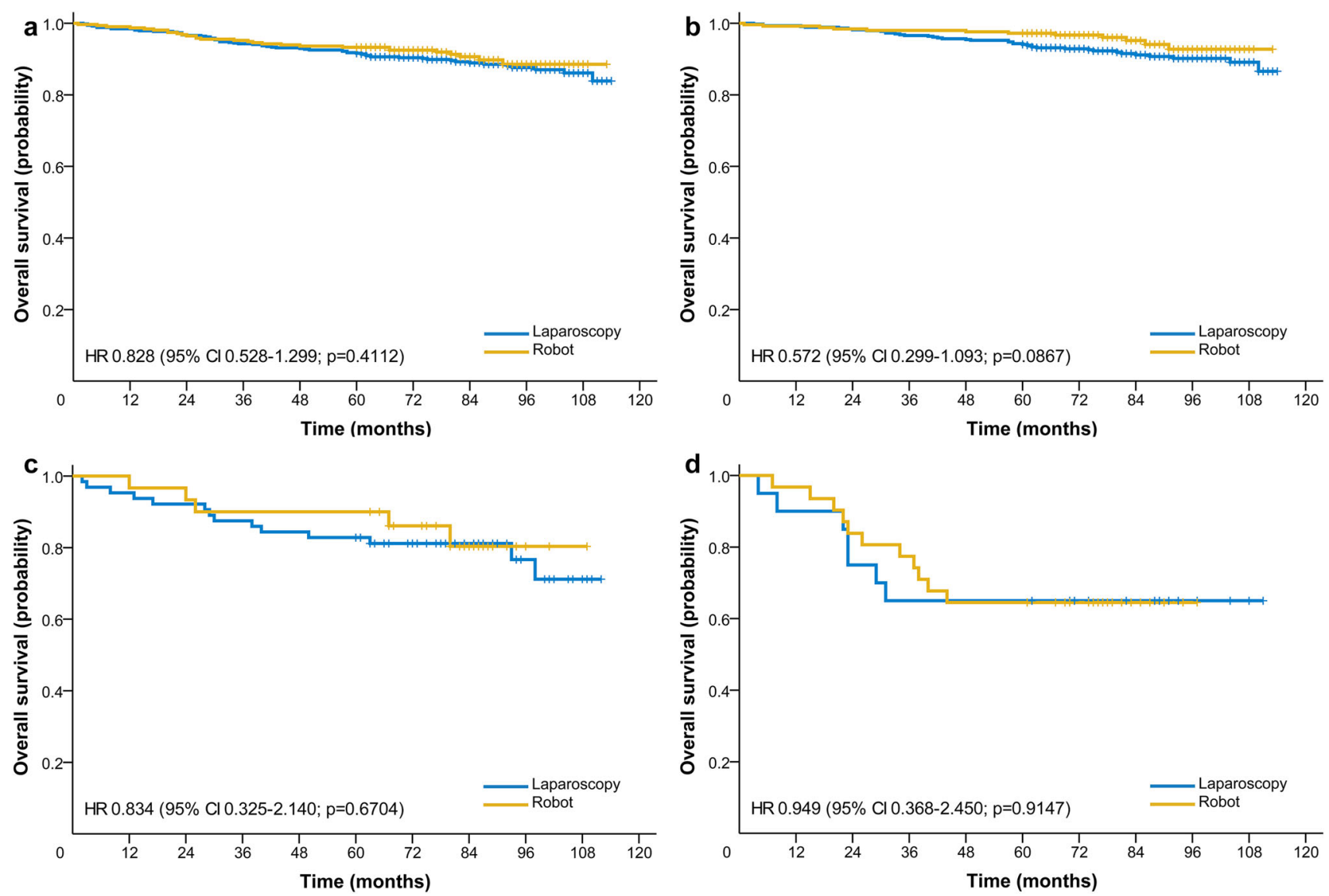

Fig. 2 Kaplan-Meier estimates of overall survival probability of all patients (a), patients with stage I (b), patients with stage II (c), and patients with stage III (d)

These did not change in survival comparisons after propensity-score matching.

The use of a robotic system is assumed to provide a technically superior operative environment for performing minimally invasive surgery $[9,13]$. However, clinically significant benefits of robotic approaches in gastric cancer surgery have yet to be sufficiently proven. In this study, the extent of lymph node dissection and the numbers of retrieved lymph nodes for the robotic and laparoscopic gastrectomies did not differ significantly, resulting in equivalent oncologic efficacy in terms of lymph node dissection between the two groups. Comparable long-term outcomes of these minimally invasive surgical modalities could be expected for procedures with same surgical extent performed in a similar operative environment. The only difference between the two approaches is the use of different surgical instruments and different imaging systems (e.g., articulating instruments and 3D imaging were used for the robotic gastrectomy surgeries); the surgical procedures themselves were the same. Unlike new chemotherapeutic agents or regimens, the objective of robotic adoption in cancer surgery is not to improve survival but to improve minimally invasive surgery performance even from the initial or early experiences and to make the surgeon easily shift from open to minimally invasive procedures [12].

While clinical trials comparing long-term outcomes between laparoscopic and open gastrectomy have yet to be published [17-19], laparoscopic gastrectomy has already prevailed and been widely accepted into clinical practice, especially for treating early gastric cancer [1]. We found that overall and relapse-free survival after robotic gastrectomy were as good as those after laparoscopic gastrectomy, supporting the oncologic safety of robotic applications in treating gastric cancer. Moreover, the overall and relapse-free survival rates in patients who underwent robotic gastrectomy in this study were comparable to those in large-scale retrospective series of laparoscopic gastrectomy [20-22]. Notably, the rate of locoregional recurrence after robotic gastrectomy was similar to that after laparoscopy. This result indicated that local control of gastric cancer with robotic applications is readily achievable. Taking the results of short- and long-term outcomes into account, the use of robotic surgery is an 

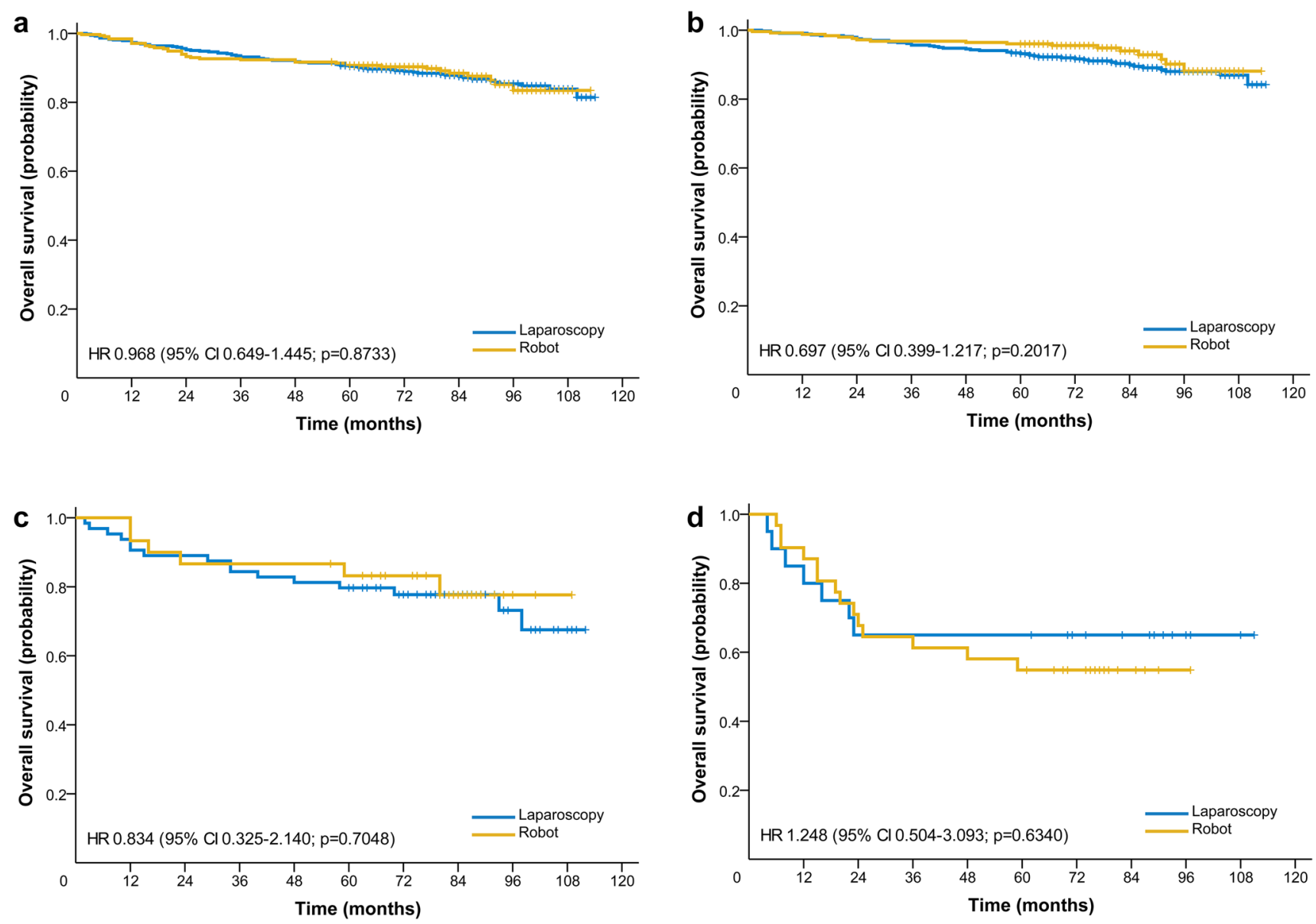

Fig. 3 Kaplan-Meier estimates of relapse-free survival probability of all patients (a), patients with stage I (b), patients with stage II (c), and patients with stage III (d)

oncologically sound approach and may be a useful alternative to laparoscopic procedures.

While it could be hypothesized that a robotic approach for more advanced gastric cancer would show superiority over a laparoscopic approach due to more precise dissection and less complications, we were unable to enroll a large number of patients with more advanced disease. Thus, we cannot expound on the superiority of robotic surgery over laparoscopic surgery in terms of survival for more advanced disease. To answer these clinical questions on the superiority of robotic surgery for more advanced disease, further experience with using the robotic approach in more advanced disease is required.

Several studies on robotic gastrectomy for gastric cancer, including prospective studies and meta-analyses of retrospective studies, have addressed its short-term outcomes [13, 23-25]. Some have suggested a potential advantage for robotic approach in reconstruction, particularly after total gastrectomy, since hand-sewn esophagojejunostomy using robotic instruments is easier than laparoscopic suturing. Moreover, even with stapled anastomosis, robotic surgery facilitates easier suturing for the closure of entry hole of linear stapler or for reinforcement, especially when the anastomosis was made in the mediastinum. However, we could not evaluate this in the present study, since we did not perform any hand-sewn anastomoses.

Results of long-term outcomes, however, have remained elusive. Studies that have reported survival outcomes have been limited by their single arm nature, small sample sizes, or short follow-up durations [26, 27]. Our study is the largest comprehensive comparative study to evaluate both short-term and long-term outcomes for robotic gastrectomy in comparison to those for laparoscopic gastrectomy. The median follow-up duration of 85 months in this study was long enough to evaluate long-term survival and recurrence. The reliability of our results was also increased by the length of the median follow-up duration; it was comparable to or longer than that of other studies of long-term outcomes of laparoscopic gastrectomy [20-22]. However, the statistical power of our study is low to be conclusive, since the number of the events such as deaths and recurrences 
Table 3 Multivariate analyses of risk factors for overall survival and relapse-free survival

\begin{tabular}{|c|c|c|c|c|c|c|c|}
\hline & \multirow[t]{2}{*}{ Frequency } & \multicolumn{3}{|c|}{ Overall survival } & \multicolumn{3}{|c|}{ Relapse-free survival } \\
\hline & & HR & $95 \% \mathrm{CI}$ & $P$ & HR & $95 \% \mathrm{CI}$ & $P$ \\
\hline Age & 837 & 1.06 & $1.03-1.08$ & $<0.001$ & 1.04 & $1.02-1.06$ & $<0.001$ \\
\hline Sex (male vs. female) & 514: 323 & 0.39 & $0.23-0.65$ & $<0.001$ & 0.44 & $0.28-0.70$ & $<0.001$ \\
\hline Size $(\leq 20 \mathrm{~mm}$ vs. $>20 \mathrm{~mm})$ & 466: 371 & 1.28 & $0.78-2.11$ & 0.324 & 1.27 & $0.81-2.00$ & 0.296 \\
\hline Histology (diff vs. undiff) & 391: 446 & 1.17 & $0.75-1.84$ & 0.493 & 1.16 & $0.76-1.75$ & 0.491 \\
\hline pT classification* & & & & 0.353 & & & 0.344 \\
\hline $\mathrm{T} 1$ & 654 & 1.00 & - & & 1.00 & - & \\
\hline $\mathrm{T} 2$ & 85 & 0.97 & $0.49-1.94$ & 0.941 & 0.91 & $0.48-1.72$ & 0.775 \\
\hline $\mathrm{T} 3$ & 48 & 1.27 & $0.62-2.60$ & 0.515 & 1.05 & $0.53-2.08$ & 0.879 \\
\hline $\mathrm{T} 4 \mathrm{a}$ & 50 & 1.91 & $0.91-4.01$ & 0.088 & 1.74 & $0.88-3.41$ & 0.109 \\
\hline pN classification* & & & & $<0.001$ & & & $<0.001$ \\
\hline No & 684 & 1.00 & - & & 1.00 & - & \\
\hline N1 & 78 & 2.51 & $1.33-4.73$ & 0.005 & 2.60 & $1.47-4.62$ & 0.001 \\
\hline $\mathrm{N} 2$ & 47 & 3.04 & $1.46-6.32$ & 0.003 & 3.55 & $1.85-6.82$ & $<0.001$ \\
\hline N3 & 28 & 10.91 & $5.15-23.10$ & $<0.001$ & 11.89 & $6.01-23.54$ & $<0.001$ \\
\hline Resection extent (STG vs. TG) & 650: 187 & 1.65 & $1.03-2.64$ & 0.039 & 1.56 & $1.02-2.39$ & 0.043 \\
\hline Type of surgery (laparoscopic vs. robotic) & 313: 524 & 0.75 & $0.46-1.21$ & 0.232 & 0.93 & $0.61-1.41$ & 0.720 \\
\hline
\end{tabular}

* UICC classification, 7 th edition

$H R$ hazard ratio, CI confidence interval, Diff differentiated, Undiff undifferentiated, STG subtotal distal gastrectomy, TG total gastrectomy

were small. Since the prognosis of the early gastric cancer patients is very good, a larger number of patients are needed to be included in the survival analyses to be confirmative to conclude similar prognosis.

At the moment, by only confirming similar prognoses, our study cannot justify the wider application of robotic approach for gastric cancer treatment due to the recognized disadvantages of longer operation time and higher cost, compared to laparoscopic surgery. Regarding longer operation time, it was also longer when laparoscopy was initially adopted. As surgeons gained experience in the technique, operation time for laparoscopic gastrectomy gradually decreased, such that they are now similar to those for open surgery [28]. Similar trends may be anticipated for robotic gastrectomy.

We did not examine quality of life after surgery or cost effectiveness; however, we expect to see future analyses of quality of life and cost effectiveness of robotic gastrectomy in comparison to laparoscopic gastrectomy from the Korean prospective multi-center comparative study [13]. Also, the data were prospectively maintained and the accumulation of patients was consecutive; however, the retrospective analysis of data from a high-volume center may limit the generalizability of our results. Results could potentially differ in low-volume centers and in patients with higher body mass index, wherein the technical superiority of a robotic approach could provide a potential clinical benefit. Notwithstanding, the similarities between the short-term results of this study compared with those of
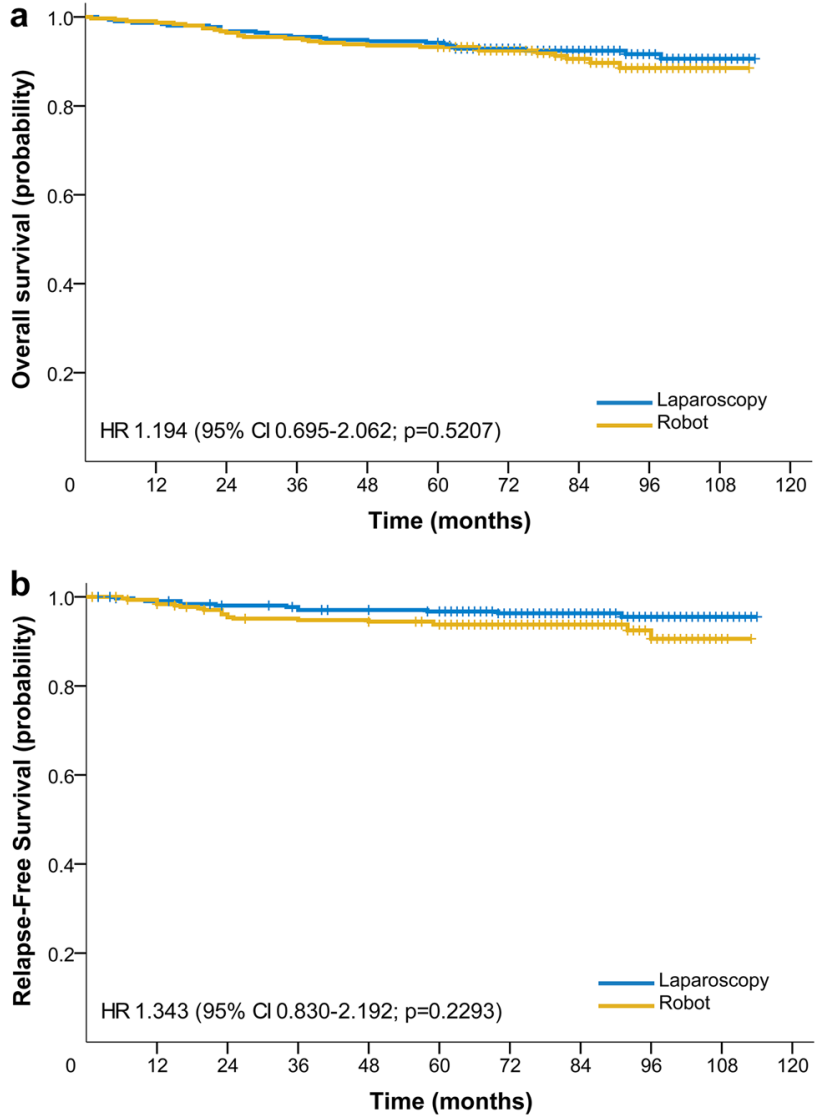

Fig. 4 Kaplan-Meier estimates of $\mathbf{a}$ overall and $\mathbf{b}$ relapse-free survival probability of propensity-score-matched cohort 
the Korean multi-center prospective study suggest that our results are not applicable to only high-volume centers.

To justify the wider use of robotic applications, further investigations should demonstrate the advantages of robotic approaches over other gastric cancer surgeries: integration of various advanced technologies can be adopted via robotic platforms without endangering the oncological outcomes of minimally invasive gastrectomy. Studies on the use of advanced technologies equipped via robotic system are warranted such as fluorescent imaging and multiple image display tool [29, 30]. Randomized controlled studies on the long-term oncologic outcomes of robotic gastrectomy are warranted to reach more definitive conclusions.

In conclusion, the technical superiority of robotic system over laparoscopy did not result in improved oncological outcomes after gastrectomy; the long-term oncological outcomes for robotic and laparoscopic gastrectomy were not different. Notwithstanding, our results may suggest that the use of robotic system in minimally invasive surgery for gastric cancer is oncologically safe.

Acknowledgements Kazutaka Obama and Yoo-Min Kim contributed equally to this work. This study was supported by a grant from the National R\&D Program for Cancer Control, Ministry of Health \& Welfare, Republic of Korea (1320270). We would like to thank Anthony Thomas Milliken, ELS (Editing Synthase, Seoul, Korea) for his assistance with editing the manuscript. We also acknowledge the assistance of BioScience Writers, LLC (Houston, TX, USA) with copyediting and correction of English language usage.

\section{Compliance and ethical standards}

Ethical standards All procedures followed were in accordance with the ethical standards of the responsible committee on human experimentation (Institutional Review Board of Severance Hospital, Yonsei University College of Medicine, Republic of Korea, approval no. 1-2010-0066) and with the Helsinki Declaration of 1964 and later versions. Informed consent or substitute for it was obtained from all patients for being included in the study.

Conflict of interest The authors declare that they have no conflict of interest.

\section{References}

1. Son T, Hyung WJ. Laparoscopic gastric cancer surgery: current evidence and future perspectives. World $\mathrm{J}$ Gastroenterol. 2016;22:727-35.

2. Zhou D, Quan Z, Wang J, Zhao M, Yang Y. Laparoscopic-assisted versus open distal gastrectomy with D2 lymph node resection for advanced gastric cancer: effect of learning curve on short-term outcomes. a meta-analysis. J Laparoendosc Adv Surg Tech A. 2014;24:139-50.

3. Moon JS, Park MS, Kim JH, Jang YJ, Park SS, Mok YJ, et al. Lessons learned from a comparative analysis of surgical outcomes of and learning curves for laparoscopy-assisted distal gastrectomy. J Gastric Cancer. 2015;15:29-38.
4. Atug F, Castle EP, Woods M, Davis R, Thomas R. Robotics in urologic surgery: an evolving new technology. Int $\mathrm{J}$ Urol. 2006;13:857-63.

5. Nifong LW, Chitwood WR, Pappas PS, Smith CR, Argenziano M, Starnes VA, et al. Robotic mitral valve surgery: a United States multicenter trial. J Thorac Cardiovasc Surg. 2005;129:1395-404.

6. Lanfranco AR, Castellanos AE, Desai JP, Meyers WC. Robotic surgery: a current perspective. Ann Surg. 2004;239:14-21.

7. Song J, Kang WH, Oh SJ, Hyung WJ, Choi SH, Noh SH. Role of robotic gastrectomy using da Vinci system compared with laparoscopic gastrectomy: initial experience of 20 consecutive cases. Surg Endosc. 2009;23:1204-11.

8. Song J, Oh SJ, Kang WH, Hyung WJ, Choi SH, Noh SH. Robotassisted gastrectomy with lymph node dissection for gastric cancer: lessons learned from an initial 100 consecutive procedures. Ann Surg. 2009;249:927-32.

9. Woo Y, Hyung WJ, Pak KH, Inaba K, Obama K, Choi SH, et al. Robotic gastrectomy as an oncologically sound alternative to laparoscopic resections for the treatment of early-stage gastric cancers. Arch Surg. 2011;146:1086-92.

10. Huang KH, Lan YT, Fang WL, Chen JH, Lo SS, Li AF, et al. Comparison of the operative outcomes and learning curves between laparoscopic and robotic gastrectomy for gastric cancer. PLoS One. 2014;9:e111499.

11. Kim HI, Park MS, Song KJ, Woo Y, Hyung WJ. Rapid and safe learning of robotic gastrectomy for gastric cancer: multidimensional analysis in a comparison with laparoscopic gastrectomy. Eur J Surg Oncol. 2014;40:1346-54.

12. Park SS, Kim MC, Park MS, Hyung WJ. Rapid adaptation of robotic gastrectomy for gastric cancer by experienced laparoscopic surgeons. Surg Endosc. 2012;26:60-7.

13. Kim HI, Han SU, Yang HK, Kim YW, Lee HJ, Ryu KW, et al. Multicenter prospective comparative study of robotic versus laparoscopic gastrectomy for gastric adenocarcinoma. Ann Surg. 2016;263:103-9.

14. Nakajima T. Gastric cancer treatment guidelines in Japan. Gastric Cancer. 2002;5:1-5.

15. Hyung WJ, Lim JS, Song J, Choi SH, Noh SH. Laparoscopic spleen-preserving splenic hilar lymph node dissection during total gastrectomy for gastric cancer. J Am Coll Surg. 2008;207:e6-11.

16. Hyung WJ, Song C, Cheong JH, Choi SH, Noh SH. Factors influencing operation time of laparoscopy-assisted distal subtotal gastrectomy: analysis of consecutive 100 initial cases. Eur J Surg Oncol. 2007;33:314-9.

17. Kim HH, Han SU, Kim MC, Hyung WJ, Kim W, Lee HJ, et al. Prospective randomized controlled trial (phase III) to comparing laparoscopic distal gastrectomy with open distal gastrectomy for gastric adenocarcinoma (KLASS 01). J Korean Surg Soc. 2013;84:123-30.

18. Hur H, Lee HY, Lee HJ, Kim MC, Hyung WJ, Park YK, et al. Efficacy of laparoscopic subtotal gastrectomy with D2 lymphadenectomy for locally advanced gastric cancer: the protocol of the KLASS-02 multicenter randomized controlled clinical trial. BMC Cancer. 2015;15:355.

19. Hu Y, Huang C, Sun Y, Su X, Cao H, Hu J, et al. Morbidity and mortality of laparoscopic versus open D2 distal gastrectomy for advanced gastric cancer: a randomized controlled trial. J Clin Oncol. 2016;34:1350-7.

20. Pak KH, Hyung WJ, Son T, Obama K, Woo Y, Kim HI, et al. Long-term oncologic outcomes of 714 consecutive laparoscopic gastrectomies for gastric cancer: results from the 7-year experience of a single institute. Surg Endosc. 2012;26:130-6.

21. Kim HH, Han SU, Kim MC, Hyung WJ, Kim W, Lee HJ, et al. Long-term results of laparoscopic gastrectomy for gastric cancer: 
a large-scale case-control and case-matched Korean multicenter study. J Clin Oncol. 2014;32:627-33.

22. Kitano S, Shiraishi N, Uyama I, Sugihara K, Tanigawa N, Japanese Laparoscopic Surgery Study G. A multicenter study on oncologic outcome of laparoscopic gastrectomy for early cancer in Japan. Ann Surg. 2007;245:68-72.

23. Tokunaga M, Sugisawa N, Kondo J, Tanizawa Y, Bando E, Kawamura T, et al. Early phase II study of robot-assisted distal gastrectomy with nodal dissection for clinical stage IA gastric cancer. Gastric Cancer. 2014;17:542-7.

24. Suda K, Man IM, Ishida Y, Kawamura Y, Satoh S, Uyama I. Potential advantages of robotic radical gastrectomy for gastric adenocarcinoma in comparison with conventional laparoscopic approach: a single institutional retrospective comparative cohort study. Surg Endosc. 2015;29:673-85.

25. Zong L, Seto Y, Aikou S, Takahashi T. Efficacy evaluation of subtotal and total gastrectomies in robotic surgery for gastric cancer compared with that in open and laparoscopic resections: a meta-analysis. PLoS One. 2014;9:e103312.

26. Coratti A, Fernandes E, Lombardi A, Di Marino M, Annecchiarico M, Felicioni L, et al. Robot-assisted surgery for gastric carcinoma: five years follow-up and beyond: a single western center experience and long-term oncological outcomes. Eur J Surg Oncol. 2015;41:1106-13.

27. Junfeng Z, Yan S, Bo T, Yingxue H, Dongzhu Z, Yongliang Z, et al. Robotic gastrectomy versus laparoscopic gastrectomy for gastric cancer: comparison of surgical performance and shortterm outcomes. Surg Endosc. 2014;28:1779-87.

28. Lee SI, Choi YS, Park DJ, Kim HH, Yang HK, Kim MC. Comparative study of laparoscopy-assisted distal gastrectomy and open distal gastrectomy. J Am Coll Surg. 2006;202:874-80.

29. Herrera-Almario G, Patane M, Sarkaria I, Strong VE. Initial report of near-infrared fluorescence imaging as an intraoperative adjunct for lymph node harvesting during robot-assisted laparoscopic gastrectomy. J Surg Oncol. 2016;113:768-70.

30. Kim YM, Baek SE, Lim JS, Hyung WJ. Clinical application of image-enhanced minimally invasive robotic surgery for gastric cancer: a prospective observational study. J Gastrointest Surg. 2013;17:304-12. 